\title{
ON KHABIBULLIN'S CONJECTURE ABOUT PAIR OF INTEGRAL INEQUALITIES
}

\section{A. BËRDËLLIMA}

\begin{abstract}
Khabibullin's conjecture is a statement about a pair of integral inequalities, where one inequality implies the other. They depend on two parameters $n \geqslant 2, n \in \mathbb{N}$, and $\alpha \in \mathbb{R}_{+}$. These inequalities were originally introduced by Khabibullin [6] in his survey regarding Paley problem in $\mathbb{C}_{n}$ and related topics about meromorphic functions. It is possible to express the inequalities in three equivalent forms. The first statement is in terms of logarithmically convex functions, the second statement is in terms of increasing functions, and the third statement is in terms of non-negative functions. In this paper we work solely with the second variant of the hypothesis. It is well established that the conjecture is true whenever $0 \leqslant \alpha \leqslant 1 / 2$ for all $n$. Several proofs exist in the literature among which one is given by the author [2] and it relates the integral inequalities with the general theory of Laplace transform. But it was not known if the statement was true when $\alpha>1 / 2$ until Sharipov [8] showed that the conjecture fails when $\alpha=2, n=2$. However the question of whether this conjecture holds for at least some $n \geqslant 2$ and $\alpha>1 / 2$ remained an open problem. In this paper we aim to solve this question. Motivated by Sharipov's approach, we develop a method of constructing a counterexample for the more general case $n \geqslant 2$ and $\alpha>1 / 2$. By an explicit counterexample we show that Khabibullin's conjecture does not hold in general.
\end{abstract}

Keywords: Khabibullin's conjecture, Khabibullin's theorem, Khabibullin's constants, integral inequalities, counterexample, plurisubharmonic function, sharp estimate.

Mathematics Subject Classification: 45A05, 31A05, 31A10, 31B05, 31B10

\section{INTRODUCTION}

A function $u$ with values in $[-\infty, \infty)$ defined in an open set $X \subset \mathbb{C}^{n}$ is called plurisubharmonic if

- $u$ is upper semi-continuous

- for arbitrary $z$ and $w \in \mathbb{C}^{n}$ the function

$$
\tau \mapsto u(z+\tau w)
$$

is subharmonic in the open subset $\mathbb{C}$ where it is defined.

In 1999 B. Khabibullin [4] conjectured the following inequality for the class of plurisubharmonic functions $u(z)$ in $\mathbb{C}^{n}$ of finite lower order $\rho$ :

$$
\liminf _{r \rightarrow \infty} \frac{M(u, r)}{T(u, r)} \leqslant P_{n}(\rho)= \begin{cases}\frac{\pi \rho}{\sin (\pi \rho)} \prod_{k=1}^{n-1}\left(1+\frac{\rho}{2 k}\right) & \text { as } \quad 0 \leqslant \rho \leqslant \frac{1}{2}, \\ \pi \rho \prod_{k=1}^{n-1}\left(1+\frac{\rho}{2 k}\right) & \text { as } \quad \rho>\frac{1}{2},\end{cases}
$$

A. Bërdëllima, On Khabibullin's COnjecture about Pair of integral inequalities.

(C) BËRDËLlIMA A. 2018.

Submitted June 24, 2017. 
where $M(u, r)=\max \left\{u(z):|z|=r, z \in \mathbb{C}^{n}\right\}, u^{+}=\max \{u(z), 0\}$ and

$$
T(u, r)=\int_{\mathbb{S}_{n}} u^{+}(r \zeta) d s_{n}(\zeta)
$$

represents the Nevanlinna characteristic of $u$. Here $\mathbb{S}_{n} \subset \mathbb{C}^{n}$ is the unit sphere, $\zeta \in \mathbb{S}_{n}$, and $d s_{n}$ is the differential of area on $\mathbb{S}_{n}$ under the normalization $\int_{\mathbb{S}_{n}} d s_{n}=1$. Conjecture $(1.1)$ is the analogue of Paley's problem for entire functions of one variable of order $\rho$, which was solved conclusively by Govorov [3]. Notice that we say a function $u$ is of finite lower order $\rho$ if

$$
\rho=\lim _{r \rightarrow \infty} \inf \frac{\log T(u, r)}{\log r} .
$$

A sufficient condition for this hypothesis to hold is proving the next statement. (For details on this sufficiency we refer to Khabibullin [5], [6] or Sharipov [7].)

Conjecture 1.1 (Khabibullin's Conjecture). Let $\alpha>1 / 2$. For any nonnegative increasing function $h(t)$ on the interval $[0, \infty)$ and for any $n \geqslant 2$ if

$$
\int_{0}^{1} \frac{h(t x)}{x}(1-x)^{n-1} d x \leqslant t^{\alpha}, \quad 0 \leqslant t<\infty
$$

then

$$
\int_{0}^{\infty} \frac{h(t)}{t} \frac{d t}{1+t^{2 \alpha}} \leqslant \frac{\pi}{2} \prod_{k=1}^{n-1}\left(1+\frac{\alpha}{k}\right) .
$$

It is also possible to express these inequalities in two other equivalent forms (Khabibullin [5], Baladai, Khabibullin [1]), but in this paper we work solely with the above stated version. For parameter values $0 \leqslant \alpha \leqslant 1 / 2$ it has been established that the integral inequalities hold true (see for instance Khabibullin [6], Sharipov [7] or Bërdëllima [2]). Regarding the same problem, by explicitly constructing a counterexample, Sharipov [8] has demonstrated that the hypothesis doesn't hold for $\alpha=2$ and $n=2$. However the question of whether this conjecture holds for at least some $n \geqslant 2$ and $\alpha>1 / 2$ remained an open problem. In this paper we aim to solve this question.

\section{PRELIMINARY DiSCUSSION}

A change of variable in inequality $(1.2) t x \mapsto x$ transforms it into the following form

$$
\int_{0}^{t} \frac{h(x)}{x}(t-x)^{n-1} d x \leqslant t^{\alpha+n-1}, \quad 0 \leqslant t<\infty .
$$

The integral inequality (2.1) can be regarded as an inequality of two functions $f(t) \leqslant g(t)$ for all $t \in[0, \infty)$, where $f(t)$ denotes the integral and $g(t):=t^{\alpha+n-1}$. On the other hand, inequality (1.3) is an inequality of quantities that are fixed for any given parameters $\alpha$ and $n$. Therefore, it is reasonable to think of getting from $(2.1)$ to $(1.3)$ through some integration over $[0, \infty)$ with some appropriate real valued function $v(t)$. If so, then there should exist some relation of $v(t)$ with the function $\phi(t):=\left(1+t^{2 \alpha}\right)^{-1}$, which appears in (1.3). To find an appropriately related function $v$, first we should study $\phi$ and in particular, its derivatives. In general, for $\phi$ we have the following representation for its derivatives

$$
\frac{d^{n}}{d t^{n}} \phi(t)=\frac{P\left(t^{\alpha}\right)}{t^{n} \cdot\left[Q\left(t^{\alpha}\right)\right]^{n+1}}, \quad n \in \mathbb{N},
$$

where $Q\left(t^{\alpha}\right)=1+t^{2 \alpha}$ and $P\left(t^{\alpha}\right)$ is a polynomial function in $t^{\alpha}$ of degree $2 n$. One can show (2.2) by using an argument of mathematical induction on $n$. An important implication of equation 
(2.2) is the growth order of $\phi^{(n)}(t)$ like $O\left(t^{-n-2 \alpha}\right)$ as $t \rightarrow \alpha^{1}$. In particular, this fact together with the inequality (2.1) imply the following vanishing limits

$$
\lim _{t \rightarrow \infty}\left|\phi^{(k)}(t)\right| \int_{0}^{t} \frac{h(x)}{x}(t-x)^{k} d x=0, \quad \forall k \in \mathbb{N} .
$$

If additionally the function $h(t)$ satisfies a restriction of the form $h(t)=o\left(t^{2 \alpha}\right)$ as $t \rightarrow \infty$, then (2.3) holds as well for $k=0$. Another key characteristic of $\phi(t)$ is its sign variation over the interval $[0, \infty)$. It is possible, though technical, to show that when $0<\alpha \leqslant 1 / 2$, the derivatives $\phi^{(n)}(t)$ are of constant sign for every $n \in \mathbb{N}$. In fact, they satisfy the following equation ${ }^{2}$

$$
\operatorname{sign} \phi^{(n)}(t)=(-1)^{n}, \quad \forall t>0, \forall n \in \mathbb{N},
$$

where $\operatorname{sign}(\cdot)$ is the usual sign function which takes the value 1 on the positive numbers, -1 on the negative numbers and 0 at the origin. Since successive integrations bring $\phi^{(n)}(t)$ to $\phi(t)$, it is justifiable to assume that there exists some relation of our sought function $v(t)$ with the derivatives of $\phi(t)$. But integrating both sides of (2.1) with $v(t)$ over $[0, \infty)$ is valid for our problem only if $v(t) \geqslant 0$ for all $t \geqslant 0$ because otherwise the order of inequality in (2.1) would reverse. At least, as $0<\alpha \leqslant 1 / 2$, we can set $v(t):=(-1)^{n} \phi^{(n)}(t)$. By 2.4 it is immediate that $v(t) \geqslant 0$. Then multiplying both sides of (2.1) by $v$ and integrating over $[0, \infty)$ yields

$$
\int_{0}^{\infty} v(t) f(t) d t \leqslant \int_{0}^{\infty} v(t) g(t) d t
$$

Using the vanishing limit conditions in $(2.3)$ and the assumption that $h(t)=o\left(t^{2 \alpha}\right)$ as $t \rightarrow \infty$, we get from integrating $n$ times by parts in $(2.5)$ the following inequality

$$
(-1)^{n} \Gamma(n) \int_{0}^{\infty} \frac{h(t)}{t}(-1)^{n} \phi(t) d t \leqslant(-1)^{n} \frac{\Gamma(\alpha+n)}{\Gamma(\alpha)} \int_{0}^{\infty} t^{\alpha-1}(-1)^{n} \phi(t) d t,
$$

where we use definitions of $f$ and $g$ and the fact that $\phi$ can be obtained from $v$ by integrating successively $n$ times. After proper simplifications in the last inequality we arrive at

$$
\int_{0}^{\infty} \frac{h(t)}{t} \phi(t) d t \leqslant \frac{\Gamma(\alpha+n)}{\Gamma(\alpha) \Gamma(n)} \int_{0}^{\infty} t^{\alpha-1} \phi(t) d t=\frac{\pi}{2 \alpha} \frac{\Gamma(\alpha+n)}{\Gamma(\alpha) \Gamma(n)}=\frac{\pi}{2} \prod_{k=1}^{n-1}\left(1+\frac{\alpha}{k}\right),
$$

which is exactly the inequality (1.3). This is but a special case of Khabibullin's theorem. One then might want to apply this method for $\alpha>1 / 2$. But in this case the derivatives of our function $\phi(t)$ are not guaranteed to be of constant sign over $[0, \infty)$ and we cannot follow a similar argument. In particular, for $n=2$ it is easy to see that $\phi^{\prime \prime}(t)$ changes sign on $[0, \infty)$. Explicit calculations show that

$$
\phi^{\prime \prime}(t)=\frac{2 \alpha t^{2 \alpha-2}\left((2 \alpha+1) t^{2 \alpha}-(2 \alpha-1)\right)}{\left(1+t^{2 \alpha}\right)^{3}}
$$

and evidently $\phi^{\prime \prime}(t)<0$ for $t \in[0, \tau)$ and $\phi^{\prime \prime}(t)>0$ for $t>\tau$, where $\tau$ is the nonzero solution of $\phi^{\prime \prime}(t)=0$ given by the formula

$$
\tau(\alpha)=\left(\frac{2 \alpha-1}{2 \alpha+1}\right)^{\frac{1}{2 \alpha}}
$$

While on one hand the failure of derivatives of $\phi$ to be of constant sign over $[0, \infty)$ invalidates the integration by parts technique, on the other hand, it provides us with an opportunity to build a counterexample. We use this sign variation of derivatives of $\phi$ to successfully set up

\footnotetext{
${ }^{1}$ With slight abuse of notation $\frac{d^{n}}{d t^{n}} \phi$ and $\phi^{(n)}(t)$ will be used interchangeably to mean the $n$-th order derivative of a function $\phi$ with respect to a real variable $t$.

${ }^{2}$ For rigorous derivations of $2.2,2.3$, and 2.4 please refer to Appendix.
} 
sufficient conditions for a counterexample to work and then we explicitly show the existence of such a counterexample.

\section{KhaBiBullin's CONJECTURE}

Before solving the problem we develop some definitions and establish some preliminary results that will be crucial in reaching the conclusion of this work.

3.1. Preliminary analysis. Studying the monotonicity of $\phi^{(n)}(t)$ over $\mathbb{R}^{+}$is very important in our task of constructing a suitable $h$ that would violate the conjecture. In the case $n=2$ it is relatively easy to characterize monotonicity of $\phi^{\prime \prime}(t)$. However, as $n>2$ getting such explicit expressions as in (2.7) becomes very difficult. By nature of $\phi(t)$, its higher order derivatives become more intricate the higher the order. In what follows for $t \in \mathbb{R}^{+}$and $\alpha>1 / 2$ we define a new function

$$
\psi_{n}(z):=P_{n}(z) \cdot\left[\frac{1}{Q(z)}\right]^{n+1} \text { with } z(t)=t^{\alpha} .
$$

It is immediate from 2.2 and 3.1 that $t^{n} \phi^{(n)}(t)=\psi_{n}(z(t))$.

Proposition 3.1. The followings are true:

1. The function $\psi_{n}(z)$ satisfies the recursive ordinary differential equation:

$$
n \psi_{n}(z)+\psi_{n+1}(z)=\alpha z \psi_{n}^{\prime}(z) \quad \text { for all } \quad z \in \mathbb{R}^{+}, n \in \mathbb{N} .
$$

2. $P_{n}(z)=z^{2} R_{n}(z)$ with $R_{n}(z)=c_{n, n-1} z^{2(n-1)}+c_{n, n-2} z^{2(n-2)}+\ldots+c_{n, 1} z^{2}+c_{n, 0}$.

3. $c_{n, k}=c_{n, k}(\alpha)$ for $k=0,1, \ldots, n-1$ are polynomials in $\alpha$. In particular,

$$
c_{n, n-1}=(-1)^{n} \prod_{k=0}^{n-1}(2 \alpha+k) \quad \text { and } \quad c_{n, 0}=-\prod_{k=0}^{n-1}(2 \alpha-k) .
$$

Proof. 1. We differentiate both sides of the equation $t^{n} \phi^{(n)}(t)=\psi_{n}(z(t))$ with respect to $t$ :

$$
n t^{n-1} \phi^{(n)}(t)+t^{n} \phi^{(n+1)}(t)=\psi_{n}^{\prime}(z) \frac{d z}{d t} .
$$

This is the same as

$$
n t^{n} \phi^{(n)}(t)+t^{n+1} \phi^{(n+1)}(t)=\psi_{n}^{\prime}(z) \frac{d z}{d t} t .
$$

Using definition of $\psi_{n}$ and $t d z / d t=\alpha t^{\alpha}=\alpha z$ gives

$$
n \psi_{n}(z)+\psi_{n+1}(z)=\alpha z \psi_{n}^{\prime}(z)
$$

2. For $n=1$ we have

$$
t \phi^{\prime}(t)=-\frac{2 \alpha t^{2 \alpha}}{\left(1+t^{2 \alpha}\right)^{2}}=-\frac{2 \alpha z^{2}}{\left(1+z^{2}\right)^{2}}=\psi_{1}(z)
$$

with $P_{1}(z)=-2 \alpha z^{2}$ and $R_{1}(z)=-2 \alpha$. Suppose the statement is true for $n=k$. Let $n=k+1$. From the differential equation for $\psi_{n}(z)$ we obtain

$$
\psi_{k+1}(z)=\alpha z \psi_{k}^{\prime}(z)-k \psi_{k}(z)=\frac{\alpha z\left[P_{k}^{\prime}(z) Q(z)-(k+1) P_{k}(z) Q^{\prime}(z)\right]-k P_{k}(z) Q(z)}{[Q(z)]^{k+2}} .
$$

By induction hypothesis $P_{k}(z)=z^{2} R_{k}(z)$ and together with $Q(z)=1+z^{2}$ this yields

$$
\psi_{k+1}(z)=\frac{z^{2}\left[\left((2 \alpha-k)\left(1+z^{2}\right)-2 \alpha(k+1) z^{2}\right) R_{k}(z)+\alpha z\left(1+z^{2}\right) R_{k}^{\prime}(z)\right]}{[Q(z)]^{k+2}} .
$$

By induction hypothesis we also have

$$
R_{k}(z)=c_{k, k-1} z^{2(k-1)}+c_{k, k-2} z^{2(k-2)}+\ldots+c_{k, 1} z^{2}+c_{k, 0},
$$


which upon termwise differentiation gives

$$
R_{k}^{\prime}(z)=2(k-1) c_{k, k-1} z^{2 k-3}+2(k-2) c_{k, k-2} z^{2 k-5}+\ldots+2 c_{k, 1} z .
$$

Substituting this in the numerator in $(3.2)$ yields

$$
\begin{aligned}
& {\left[(2 \alpha-k)\left(1+z^{2}\right)-2 \alpha(k+1) z^{2}\right] R_{k}(z)+\alpha z\left(1+z^{2}\right) R_{k}^{\prime}(z)} \\
& \quad=\left[(2 \alpha-k)\left(1+z^{2}\right)-2 \alpha(k+1) z^{2}\right] \sum_{i=0}^{k-1} c_{k, i} z^{2 i}+2 \alpha z\left(1+z^{2}\right) \sum_{i=1}^{k-1} i c_{k, i} z^{2 i-1} \\
& =\sum_{i=0}^{k-1}(2 \alpha(i+1)-k) c_{k, i} z^{2 i}+\sum_{i=0}^{k-1}-(2 \alpha(k-i)+k) c_{k, i} z^{2 i+2} \\
& =(2 \alpha-k) c_{k, 0}+(-1)(2 \alpha+k) c_{k, k-1} z^{2 k} \\
& \quad+\sum_{i=1}^{k-1}\left[(2 \alpha(i+1)-k) c_{k, i}-2 \alpha(k+1-i) c_{k, i-1}\right] z^{2 i} \\
& =c_{k+1,0}+c_{k+1, k} z^{2 k}+\sum_{i=1}^{k-1} c_{k+1, i} z^{2 i}:=R_{k+1}(z) .
\end{aligned}
$$

where we have defined

$$
c_{k+1, i}= \begin{cases}(2 \alpha-k) c_{k, 0} & \text { as } \quad i=0, \\ (-1)(2 \alpha+k) c_{k, k-1} & \text { as } \quad i=k, \\ (2 \alpha(i+1)-k) c_{k, i}-2 \alpha(k+1-i) c_{k, i-1} & \text { as } \quad i=1,2, \ldots, k-1 .\end{cases}
$$

This completes the proof of (ii).

3. The identities for $c_{k, 0}$ and $c_{k, k-1}$ follow directly the definition of $c_{k, i}$ for $i=0$ and $i=k-1$ with initial condition $c_{1,0}=-2 \alpha$. Also using this initial condition, one can show by induction that $c_{k, i}=c_{k, i}(\alpha)$ are polynomials in $\alpha$ for all $k \in \mathbb{N}$ and $i=0,1, \ldots, k-1$. This is implied by (3.3) as polynomials form a ring closed under addition and multiplication.

Definition 3.1. Let $T \subseteq \mathbb{R}$. A real valued function $f: T \rightarrow \mathbb{R}$ is called proper on $T$ if $f$ is identically different from zero on every compact subset $S \subseteq T$.

Notice that for any $n \geqslant 2$ the function $\phi^{(n)}(t)$ is proper on $\mathbb{R}^{+}$. Otherwise there would exist some compact set $S \subseteq \mathbb{R}^{+}$with $P\left(t^{\alpha}\right) \equiv 0$ on $S$. Since $P$ is a polynomial function then it would follow that $P$ is identically equal to zero on all $\mathbb{R}$. But this contradicts $(2.2)$, which states that $P$ is a nonconstant polynomial function.

Definition 3.2. Let $T \subseteq \mathbb{R}$ and $f: T \rightarrow \mathbb{R}$ be a continuous real valued proper function. The sign variation set of $f$, denoted by $\mathcal{T}_{f}$, is defined as

$$
\mathcal{T}_{f}:=\{t \in T: \exists r>0, \forall \delta \in(0, r] f(t+\delta) \neq 0 \& \operatorname{sign}(f(t+\delta))=-\operatorname{sign}(f(t-\delta))\} .
$$

In simple words, $T_{f}$ is the set of points where the function $f$ changes sign. Another way to think about the sign variation set of a continuous proper function is in terms of its zeros. Notice that if $\tau \in \mathcal{T}_{f}$ then $f(\tau)=0$ by continuity of $f$. In fact $\mathcal{T}_{f}$ coincides with the set of zeros of $f$ of odd order (multiplicity). We are interested now in the sign variation set of the function $\phi^{(n)}(t)$ over $\mathbb{R}^{+}$that we denote by $\mathcal{T}_{\phi^{(n)}}$. When $n=2, \phi^{\prime \prime}(t)<0$ for all $t<\tau$ and $\phi^{\prime \prime}(t)>0$ for all $t \in(\tau, \infty)$ with $\tau$ given by $(2.7)$. This implies $\mathcal{T}_{\phi^{\prime \prime}}=\{\tau\} \neq \emptyset$. For $n>2$ the higher order derivatives of $\phi(t)$ become more complicated and it is not clear whether $\mathcal{T}_{\phi^{(n)}}$ is nonempty or not. The next statement remedies this issue.

Proposition 3.2. For any $n \geqslant 2$ and $n \in \mathbb{N}$ the sign variation set $\mathcal{T}_{\phi^{(n)}}$ is nonempty. Moreover, $\mathcal{T}_{\phi(n)}$ is finite. 
Proof. Suppose that there exists $n \in \mathbb{N}$ such that $\mathcal{T}_{\phi(n)}=\emptyset$ and without loss of generality let it be the smallest such $n$. Clearly, $n>2$ as we know by construction that $\mathcal{T}_{\phi^{\prime \prime}} \neq \emptyset$ as $n=2$. Now for any $\tau \in \mathcal{T}_{\phi^{(n-1)}}$ we have

$$
\phi^{(n-1)}(\tau)=0 \Rightarrow \tau^{n-1} \phi^{(n-1)}(\tau)=0 \Rightarrow \psi_{n-1}\left(z^{*}\right)=0 \text { with } \quad z^{*}:=\tau^{\alpha} .
$$

By proposition 3.1 (1) it follows that

$$
\alpha z^{*} \psi_{n-1}^{\prime}\left(z^{*}\right)=n \psi_{n-1}\left(z^{*}\right)+\psi_{n}\left(z^{*}\right)=\psi_{n}\left(z^{*}\right) .
$$

Since $\mathcal{T}_{\phi^{(n)}}=\emptyset$, this yields that $\phi^{(n)}(t) \geqslant 0$ or $\phi^{(n)}(t) \leqslant 0$ for all $t \in \mathbb{R}^{+}$. Without loss of generality we assume that $\phi^{(n)}(t) \geqslant 0$ for all $t \in \mathbb{R}^{+}$. This is equivalent to $\psi_{n}(z) \geqslant 0$ for all $z \geqslant 0$ by definition of $\psi_{n}$ in $(3.1)$. In particular, for $t=\tau$ we have $\phi^{(n)}(\tau) \geqslant 0$ and this implies $\psi_{n}\left(z^{*}\right) \geqslant 0$. Therefore, we have

$$
\alpha z^{*} \psi_{n-1}^{\prime}\left(z^{*}\right)=\psi_{n}\left(z^{*}\right) \geqslant 0 \Rightarrow \psi_{n-1}^{\prime}\left(z^{*}\right) \geqslant 0 .
$$

On the other hand, $Q\left(z^{*}\right)=1+\left(z^{*}\right)^{2}>0$. Since $\psi_{n-1}\left(z^{*}\right)=0$ then it follows that $P_{n-1}\left(z^{*}\right)=0$. Explicit differentiation of $\psi_{n-1}(z)$ and its evaluation at $z^{*}$ yields

$$
\psi_{n-1}^{\prime}\left(z^{*}\right)=\frac{P_{n-1}^{\prime}\left(z^{*}\right) Q\left(z^{*}\right)-n P_{n-1}\left(z^{*}\right) Q^{\prime}\left(z^{*}\right)}{\left[Q\left(z^{*}\right)\right]^{n+1}}=\frac{P_{n-1}^{\prime}\left(z^{*}\right) Q^{\prime}\left(z^{*}\right)}{\left[Q\left(z^{*}\right)\right]^{n+1}}
$$

and (3.4) and (3.5) imply $P_{n-1}^{\prime}\left(z^{*}\right) \geqslant 0$. Note that $P_{n-1}(z)$ is a polynomial function and hence is continuously differentiable everywhere on $\mathbb{R}$. Now suppose that there exists $z^{* *}>z^{*}$ with $P_{n-1}\left(z^{* *}\right)=0$, then by Rolle's theorem ${ }^{1}$ there exists $w \in\left(z^{*}, z^{* *}\right)$ such that $P_{n-1}^{\prime}(w)=0$. Moreover, by the fact that $P_{n-1}^{\prime}\left(z^{*}\right) \geqslant 0$ it must be the case that $P_{n-1}(w)>0$, which in turn implies $\psi_{n-1}(w)>0$ and $\psi_{n-1}^{\prime}(w)<0$. From the differential equation we then get

$$
0>\alpha w \psi_{n-1}^{\prime}(w)=n \psi_{n-1}(w)+\psi_{n}(w)>\psi_{n}(w) \Rightarrow \psi_{n}(w)<0 .
$$

However, this contradicts our assumption that $\psi_{n}(z) \geqslant 0$ everywhere on $\mathbb{R}^{+}$. Therefore, an arbitrarily chosen $\tau$ is the only element of $\mathcal{T}_{\phi^{(n-1)}}$. Since $P_{n-1}^{\prime}\left(z^{*}\right) \geqslant 0$, this implies

$$
\lim _{z \rightarrow \infty} \frac{P_{n-1}(z)}{z^{2(n-1)}}>0 \Rightarrow \lim _{z \rightarrow \infty} \frac{R_{n-1}(z)}{z^{2(n-2)}}>0 \Leftrightarrow c_{n-1, n-2}>0 \Leftrightarrow(-1)^{n-1} \prod_{k=0}^{n-2}(2 \alpha+k)>0 .
$$

On the other hand, by assumption we have $\phi^{(n)}(t) \geqslant 0$ for all $t \in \mathbb{R}^{+}$. This in turn implies $\psi_{n}(z) \geqslant 0$ for all $z>0$. In particular, we have $\lim _{z \rightarrow \infty} \psi_{n}(z) \geqslant 0$, which then yields

$$
\lim _{z \rightarrow \infty} \frac{P_{n}(z)}{z^{2 n}} \geqslant 0 \Rightarrow \lim _{z \rightarrow \infty} \frac{R_{n}(z)}{z^{2(n-1)}} \geqslant 0 \Leftrightarrow c_{n, n-1} \geqslant 0 \Leftrightarrow(-1)^{n} \prod_{k=0}^{n-1}(2 \alpha+k) \geqslant 0 .
$$

However, (3.6) and 3.7) cannot hold simultaneously. Therefore, we arrive at a contradiction. This establishes $\mathcal{T}_{\phi^{(n)}} \neq \emptyset$ for any $n \geqslant 2$ and $n \in \mathbb{N}$. The set $\mathcal{T}_{\phi^{(n)}}$ is finite for any $n \geqslant 2$ as it is implied by the fact that each element $\tau \in \mathcal{T}_{\phi^{(n)}}$ is vis-a-vis related to a zero $z^{*}$ of odd multiplicity of the polynomial $R_{n}(z)$ through the relation $z^{*}=\tau^{\alpha}$. By the fundamental theorem of algebra $2^{2} R_{n}(z)$ has at most $\operatorname{deg}\left(R_{n}\right)$ zeros, not necessarily distinct. Therefore, $\left|\mathcal{T}_{\phi^{(n)}}\right| \leqslant \operatorname{deg}\left(R_{n}\right)<\infty$

\footnotetext{
${ }^{1}$ Rolle's theorem: If $f:[a, b] \rightarrow \mathbb{R}$ is differentiable on $(a, b)$ and continuous on $[a, b]$ with $f(a)=f(b)$ then there exists $c \in(a, b)$ such that $f^{\prime}(c)=0$.

${ }^{2}$ The fundamental theorem of algebra: Every nonzero, single variable, polynomial of degree $n \geqslant 0$ with complex coefficients has at most $n$ complex roots (not necessarily distinct).
} 
3.2. Sufficient conditions. Now let $0<\tau_{1}<\tau_{2}<\ldots<\tau_{m}<\infty$ be an ordering of the elements of $\mathcal{T}_{\phi^{(n)}}$ for some $n \geqslant 2$ and $m=\left|\mathcal{T}_{\phi^{(n)}}\right|$. We partition $\mathbb{R}^{+}$into disjoint intervals:

$$
\mathbb{R}^{+}=\left(0, \tau_{1}\right] \cup\left(\tau_{1}, \tau_{2}\right] \cup \ldots \cup\left(\tau_{m-1}, \tau_{m}\right] \cup\left(\tau_{m}, \infty\right) .
$$

Define the following sets: $\mathcal{T}_{n}^{+}:=\left\{t \in \mathbb{R}^{+}: \operatorname{sign}\left(\phi^{n}(t)\right)=(-1)^{n+1}\right\}$ and the index set $\mathcal{I}_{n}^{+}:=$ $\left\{i \in \mathbb{N}: \operatorname{sign}\left(\phi^{n}(t)\right)=(-1)^{n+1}, \forall t \in\left(\tau_{i-1}, \tau_{i}\right]\right\}$. Since $\operatorname{sign}\left(\phi^{(n)}(t)\right)=\operatorname{sign}\left(c_{n, n-1}\right)=(-1)^{n}$ by the proof of the last proposition for all $t>\tau_{m}$ we have $\left(\tau_{m}, \infty\right) \notin \mathcal{T}_{n}^{+}$and so we get

$$
\mathcal{T}_{n}^{+}=\bigcup_{i \in \mathcal{I}_{n}^{+}}\left(\tau_{i-1}, \tau_{i}\right]
$$

Fix any $n \geqslant 2, n \in \mathbb{N}$ and $\alpha>1 / 2$. Next we need to construct a pair of functions $\{f, h\}$ with $h$ being continuously differentiable on $\mathbb{R}^{+}$. This pair of functions should satisfy the following relation for all $t \in \mathbb{R}^{+}$

$$
f(t)=\int_{0}^{t} \frac{h(x)}{x}(t-x)^{n-1} d x \quad \text { or equivalently } h(t)=\frac{1}{\Gamma(n)} t \frac{d^{n}}{d t^{n}} f(t)
$$

Let $\left\{\eta_{i}\right\}_{i \in \mathcal{I}_{n}^{+}}$be a family of functions $\eta_{i}:\left(\tau_{i-1}, \tau_{i}\right] \rightarrow[0,1]$ such that $\eta_{i}(t)=0$ for all $t \notin\left(\tau_{i-1}, \tau_{i}\right]$ and $\eta_{i}$ is sufficiently smooth (derivatives of orders at least $n+1$ exist) on $\left(\tau_{i-1}, \tau_{i}\right)$ for all $i \in \mathcal{I}_{n}^{+}$. Define function $f$ in the following way

$$
f(t)=\left\{\begin{array}{lll}
t^{\alpha+n-1}\left(1-\varepsilon \sum_{i \in \mathcal{I}_{n}^{+}} \eta_{i}(t)\right) & \text { as } & t \in \mathcal{T}_{n}^{+} \\
t^{\alpha+n-1} & \text { as } \quad t \in \mathbb{R}^{+} \backslash \mathcal{T}_{n}^{+}
\end{array}\right.
$$

Then $f(t)$ satisfies the inequality $0 \leqslant f \leqslant t^{\alpha+n-1}$ whenever $0 \leqslant \varepsilon \leqslant 1$. The family of functions $\left\{\eta_{i}\right\}_{i \in \mathcal{I}_{n}^{+}}$should satisfy conditions derived from the properties of $h$. Differentiability of $h$ and relation (3.8) imply that $f$ must have continuous derivatives at least to the order $n+1$. This leads us to the following limits

$$
\lim _{t \rightarrow \tau_{i}^{-}} \frac{d^{k}}{d t^{k}}\left[t^{\alpha+n-1} \eta_{i}(t)\right]=0, \quad \lim _{t \rightarrow \tau_{i-1}^{+}} \frac{d^{k}}{d t^{k}}\left[t^{\alpha+n-1} \eta_{i}(t)\right]=0, \quad k=1,2, \ldots, n+1, \quad i \in \mathcal{I}_{n}^{+} .
$$

Condition (3.10) illustrates the fact that the left and the right limits at $t=\tau_{i}$ for $i \in \mathcal{I}_{n}^{+}$of derivatives of $f$ up to order $n+1$ should coincide. The increasing and nonnegativity property of $h$ lead us to the following conditions

$$
\begin{aligned}
& \frac{1}{\Gamma(n)} t \frac{d^{n}}{d t^{n}} f(t) \geqslant 0, \quad t \in \mathcal{T}_{n}^{+} \\
& \frac{1}{\Gamma(n)} \frac{d^{n}}{d t^{n}} f(t)+\frac{1}{\Gamma(n)} t \frac{d^{n+1}}{d t^{n+1}} f(t) \geqslant 0, \quad t \in \mathcal{T}_{n}^{+} .
\end{aligned}
$$

We consider only $\mathcal{T}_{n}^{+}$because (3.11) and (3.12) trivially hold on $\mathbb{R}^{+} \backslash \mathcal{T}_{n}^{+}$by definition of $f$ in (3.9). From the same definition and (3.8) one can get also an explicit piecewise formulation of $h$ :

$$
h(t):=\frac{1}{\Gamma(n)} t \frac{d^{n}}{d t^{n}} f(t)= \begin{cases}C(n, \alpha) t^{\alpha}-\frac{\varepsilon}{\Gamma(n)} t \sum_{i \in \mathcal{I}_{n}^{+}} \frac{d^{n}}{d t^{n}}\left[t^{\alpha+n-1} \eta_{i}(t)\right] & \text { as } \quad t \in \mathcal{T}_{n}^{+} \\ C(n, \alpha) t^{\alpha} & \text { as } \quad t \in \mathbb{R}^{+} \backslash \mathcal{T}_{n}^{+},\end{cases}
$$

where $C(n, \alpha):=\frac{\Gamma(n+\alpha)}{\Gamma(\alpha) \Gamma(n)}$. We consider the integral

$$
J_{\varepsilon}(\alpha):=\int_{\mathbb{R}^{+}} \frac{h(t)}{t} \phi(t) d t,
$$


Using the piecewise representation of $h(t)$ in $(3.13)$, this follows that

$$
\begin{aligned}
J_{\varepsilon}(\alpha) & =-\frac{\varepsilon}{\Gamma(n)} \int_{\mathcal{T}_{n}^{+}} \sum_{i \in \mathcal{I}_{n}^{+}} \frac{d^{n}}{d t^{n}}\left[t^{\alpha+n-1} \eta_{i}(t)\right] \phi(t) d t+C(n, \alpha) \int_{\mathbb{R}^{+}} t^{\alpha-1} \phi(t) d t \\
& =\sum_{i \in \mathcal{I}_{n}^{+}}-\frac{\varepsilon}{\Gamma(n)} \int_{\mathcal{T}_{n}^{+}} \frac{d^{n}}{d t^{n}}\left[t^{\alpha+n-1} \eta_{i}(t)\right] \phi(t) d t+\int_{\mathbb{R}^{+}} \frac{\widetilde{h}_{n}(t)}{t} \phi(t) d t
\end{aligned}
$$

where $\widetilde{h}_{n}(t)=C(n, \alpha) t^{\alpha}$. We integrate $n$-times by parts in the integral expression inside the sum. Notice that from conditions in 3.10 its integrand vanishes on the boundary $\partial \mathcal{T}_{n}^{+}=\mathcal{T}_{\phi^{(n)}}$. Therefore, we arrive at the following expression

$$
J_{\varepsilon}(\alpha)=(-1)^{n+1} \sum_{i \in \mathcal{I}_{n}^{+}} \frac{\varepsilon}{\Gamma(n)} \int_{\mathcal{T}_{n}^{+}} t^{\alpha+n-1} \eta_{i}(t) \frac{d^{n}}{d t^{n}} \phi(t) d t+\int_{\mathbb{R}^{+}} \frac{\widetilde{h}_{n}(t)}{t} \phi(t) d t>\int_{\mathbb{R}^{+}} \frac{\widetilde{h}_{n}(t)}{t} \phi(t) d t
$$

whenever

$$
(-1)^{n+1} \frac{d^{n}}{d t^{n}} \phi(t) \geqslant 0, \quad t \in \mathcal{T}_{n}^{+}
$$

This inequality is implied by the definition of $\mathcal{T}_{n}^{+}$. Therefore, the second integral inequality would violate the conjectured upper estimate. Naturally, one raises the question of whether a family of functions $\left\{\eta_{i}\right\}_{i \in \mathcal{I}_{n}^{+}}$satisfying $3.10-3.12$ exists. This will be shown in the next section.

3.3. A general counterexample. Let $n \geqslant 2$. Consider the collection of functions $\left\{\eta_{i}\right\}_{i \in \mathcal{I}_{n}^{+}}$ given by

$$
\eta_{i}(t)= \begin{cases}{\left[\cos \left(\frac{\pi t}{2 \tau_{i-1}}\right) \cos \left(\frac{\pi t}{2 \tau_{i}}\right)\right]^{2 n}} & \text { as } \quad t \in\left(\tau_{i-1}, \tau_{i}\right] \\ 0 & \text { as } \quad t \notin\left(\tau_{i-1}, \tau_{i}\right]\end{cases}
$$

with the convention that if $1 \in \mathcal{I}_{n}^{+}$as $\eta_{1}(t)=\cos ^{2 n}\left(\frac{\pi t}{2 \tau_{1}}\right)$ for all $t \in\left(0, \tau_{1}\right]$ and zero otherwise. By definition in (3.15), the function $\eta_{i}$ is smooth (infinitely differentiable) on $\left(\tau_{i-1}, \tau_{i}\right)$ for all $i \in \mathcal{I}_{n}^{+}$. On the other hand, by Leibniz's formula we have for $k=0,1, . ., n+1$

$$
\begin{aligned}
& \lim _{t \rightarrow \tau_{i-1}^{+}} \frac{d^{k}}{d t^{k}}\left[t^{\alpha+n-1} \eta_{i}(t)\right]=\sum_{j=0}^{k}\left(\begin{array}{l}
k \\
j
\end{array}\right) \lim _{t \rightarrow \tau_{i-1}^{+}} \frac{d^{j}}{d t^{j}} t^{\alpha+n-1} \cos ^{2 n}\left(\frac{\pi t}{2 \tau_{i}}\right) \lim _{t \rightarrow \tau_{i-1}^{+}} \frac{d^{k-j}}{d t^{k-j}} \cos ^{2 n}\left(\frac{\pi t}{2 \tau_{i-1}}\right)=0, \\
& \lim _{t \rightarrow \tau_{i}^{-}} \frac{d^{k}}{d t^{k}}\left[t^{\alpha+n-1} \eta_{i}(t)\right]=\sum_{j=0}^{k}\left(\begin{array}{l}
k \\
j
\end{array}\right) \lim _{t \rightarrow \tau_{i}^{-}} \frac{d^{j}}{d t^{j}} t^{\alpha+n-1} \cos ^{2 n}\left(\frac{\pi t}{2 \tau_{i-1}}\right) \lim _{t \rightarrow \tau_{i}^{-}} \frac{d^{k-j}}{d t^{k-j}} \cos ^{2 n}\left(\frac{\pi t}{2 \tau_{i}}\right)=0 .
\end{aligned}
$$

Hence, the limit conditions in (3.10) are fulfilled. Next we need to check (3.11), which by piecewise representation of $h$ is equivalent to

$$
C(n, \alpha) t^{\alpha} \geqslant \frac{\varepsilon}{\Gamma(n)} t \sum_{i \in \mathcal{I}_{n}^{+}} \frac{d^{n}}{d t^{n}}\left[t^{\alpha+n-1} \eta_{i}(t)\right], \quad t \in \mathcal{T}_{n}^{+}
$$

Another application of Lebniz's formula on the last expression yields

$$
\begin{aligned}
\frac{d^{n}}{d t^{n}}\left[t^{\alpha+n-1} \eta_{i}(t)\right] & =\sum_{j=0}^{n}\left(\begin{array}{c}
n \\
j
\end{array}\right) \frac{d^{j}}{d t^{j}} t^{\alpha+n-1} \frac{d^{n-j}}{d t^{n-j}} \eta_{i}(t) \\
& =t^{\alpha-1} \sum_{j=0}^{n}\left(\begin{array}{c}
n \\
j
\end{array}\right) \frac{\Gamma(\alpha+n)}{\Gamma(\alpha+n-j)} t^{n-j} \frac{d^{n-j}}{d t^{n-j}} \eta_{i}(t) .
\end{aligned}
$$


Relabelling $j \mapsto n-j$ in 3.17 and using the identity $\left(\begin{array}{c}n \\ j\end{array}\right)=\left(\begin{array}{c}n \\ n-j\end{array}\right)$, we rewrite (3.16) as

$$
C(n, \alpha) \geqslant \varepsilon \sum_{i \in \mathcal{I}_{n}^{+}} \sum_{j=0}^{n}\left(\begin{array}{l}
n \\
j
\end{array}\right) \frac{\Gamma(\alpha+n)}{\Gamma(n) \Gamma(\alpha+j)} t^{j} \frac{d^{j}}{d t^{j}} \eta_{i}(t) \quad \forall t \in \mathcal{T}_{n}^{+}
$$

for some suitably chosen $\varepsilon \in[0,1]$. In fact, using the definition of $C(\alpha, n)$ one can simplify even further the last inequality into the following equivalent form

$$
1 \geqslant \varepsilon \sum_{i \in \mathcal{I}_{n}^{+}} \sum_{j=0}^{n}\left(\begin{array}{l}
n \\
j
\end{array}\right) \frac{\Gamma(\alpha)}{\Gamma(\alpha+j)} t^{j} \frac{d^{j}}{d t^{j}} \eta_{i}(t), \quad t \in \mathcal{T}_{n}^{+} .
$$

Similarly using the piecewise representation of $h$, we rewrite condition 3.12 as

$$
\alpha C(n, \alpha) t^{\alpha-1} \geqslant \frac{\varepsilon}{\Gamma(n)} \sum_{i \in \mathcal{I}_{n}^{+}} \frac{d^{n}}{d t^{n}}\left[t^{\alpha+n-1} \eta_{i}(t)\right]+\frac{\varepsilon}{\Gamma(n)} t \sum_{i \in \mathcal{I}_{n}^{+}} \frac{d^{n+1}}{d t^{n+1}}\left[t^{\alpha+n-1} \eta_{i}(t)\right], \quad t \in \mathcal{T}_{n}^{+} .
$$

Again by Leibniz's formula we have

$$
\begin{aligned}
\frac{d^{n+1}}{d t^{n+1}}\left[t^{\alpha+n-1} \eta_{i}(t)\right] & =\sum_{j=0}^{n+1}\left(\begin{array}{c}
n+1 \\
j
\end{array}\right) \frac{d^{j}}{d t^{j}} t^{\alpha+n-1} \frac{d^{n+1-j}}{d t^{n+1-j}} \eta_{i}(t) \\
& =t^{\alpha-2} \sum_{j=0}^{n+1}\left(\begin{array}{c}
n+1 \\
j
\end{array}\right) \frac{\Gamma(\alpha+n)}{\Gamma(\alpha+n-j)} t^{n+1-j} \frac{d^{n+1-j}}{d t^{n+1-j}} \eta_{i}(t) \\
& =t^{\alpha-2} \sum_{j=0}^{n+1}\left(\begin{array}{c}
n+1 \\
j
\end{array}\right) \frac{\Gamma(\alpha+n)}{\Gamma(\alpha+j-1)} t^{j} \frac{d^{j}}{d t^{j}} \eta_{i}(t) .
\end{aligned}
$$

Using 3.20 in conjunction with 3.17$)$ we can write 3.19 as follows

$$
\begin{aligned}
\alpha C(n, \alpha) t^{\alpha-1} \geqslant & \varepsilon t^{\alpha-1}\left[\sum_{i \in \mathcal{I}_{n}^{+}} \sum_{j=0}^{n}\left(\begin{array}{c}
n \\
j
\end{array}\right) \frac{\Gamma(\alpha+n)}{\Gamma(n) \Gamma(\alpha+j)} t^{j} \frac{d^{j}}{d t^{j}} \eta_{i}(t)\right. \\
& \left.+\sum_{i \in \mathcal{I}_{n}^{+}} \sum_{j=0}^{n+1}\left(\begin{array}{c}
n+1 \\
j
\end{array}\right) \frac{\Gamma(\alpha+n)}{\Gamma(n) \Gamma(\alpha+j-1)} t^{j} \frac{d^{j}}{d t^{j}} \eta_{i}(t)\right], \quad t \in \mathcal{T}_{n}^{+} .
\end{aligned}
$$

Since $\alpha>0$, after proper simplifications we have the inequality in its final form

$$
1 \geqslant \frac{\varepsilon}{\alpha} \sum_{i \in \mathcal{I}_{n}^{+}}\left[\sum_{j=0}^{n}\left(\begin{array}{c}
n \\
j
\end{array}\right) \frac{\Gamma(\alpha)}{\Gamma(\alpha+j)} t^{j} \frac{d^{j}}{d t^{j}} \eta_{i}(t)+\sum_{j=0}^{n+1}\left(\begin{array}{c}
n+1 \\
j
\end{array}\right) \frac{\Gamma(\alpha)}{\Gamma(\alpha+j-1)} t^{j} \frac{d^{j}}{d t^{j}} \eta_{i}(t)\right], \quad t \in \mathcal{T}_{n}^{+} .
$$

For each $i \in \mathcal{I}_{n}^{+}, \eta_{i}(t)$ is smooth on the bounded interval $\left(\tau_{i-1}, \tau_{i}\right)$, and the supremum of each derivative of $\eta_{i}$ exists on this interval and is finite. For brevity denote the supremum of the $j$-th derivative of $\eta_{i}, j=0,1, \ldots, n$, by

$$
\eta_{i, j}^{*}:=\sup _{t \in\left(\tau_{i-1}, \tau_{i}\right)}\left\{\left|\frac{d^{j}}{d t^{j}} \eta_{i}(t)\right|\right\} .
$$

We get the estimates:

$$
\left|\sum_{i \in \mathcal{I}_{n}^{+}} \sum_{j=0}^{n}\left(\begin{array}{l}
n \\
j
\end{array}\right) \frac{\Gamma(\alpha)}{\Gamma(\alpha+j)} t^{j} \frac{d^{j}}{d t^{j}} \eta_{i}(t)\right| \leqslant \sum_{i \in \mathcal{I}_{n}^{+}} \sum_{j=0}^{n}\left(\begin{array}{c}
n \\
j
\end{array}\right) \frac{\Gamma(\alpha)}{\Gamma(\alpha+j)} \tau_{i}^{j} \eta_{i, j}^{*}<\infty, \quad t \in \mathcal{T}_{n}^{+}
$$




$$
\begin{aligned}
& \left|\sum_{i \in \mathcal{I}_{n}^{+}}\left[\sum_{j=0}^{n}\left(\begin{array}{c}
n \\
j
\end{array}\right) \frac{\Gamma(\alpha)}{\Gamma(\alpha+j)} t^{j} \frac{d^{j}}{d t^{j}} \eta_{i}(t)+\sum_{j=0}^{n+1}\left(\begin{array}{c}
n+1 \\
j
\end{array}\right) \frac{\Gamma(\alpha)}{\Gamma(\alpha+j-1)} t^{j} \frac{d^{j}}{d t^{j}} \eta_{i}(t)\right]\right| \\
& \leqslant \sum_{i \in \mathcal{I}_{n}^{+}}\left[\sum_{j=0}^{n}\left(\begin{array}{c}
n \\
j
\end{array}\right) \frac{\Gamma(\alpha)}{\Gamma(\alpha+j)} t^{j}\left|\frac{d^{j}}{d t^{j}} \eta_{i}(t)\right|+\sum_{j=0}^{n+1}\left(\begin{array}{c}
n+1 \\
j
\end{array}\right) \frac{\Gamma(\alpha)}{\Gamma(\alpha+j-1)} t^{j}\left|\frac{d^{j}}{d t^{j}} \eta_{i}(t)\right|\right] \\
& \leqslant \sum_{i \in \mathcal{I}_{n}^{+}}\left[\sum_{j=0}^{n}\left(\begin{array}{c}
n \\
j
\end{array}\right) \frac{\Gamma(\alpha)}{\Gamma(\alpha+j)} \tau_{i}^{j} \eta_{i, j}^{*}+\sum_{j=0}^{n+1}\left(\begin{array}{c}
n+1 \\
j
\end{array}\right) \frac{\Gamma(\alpha)}{\Gamma(\alpha+j-1)} \tau_{i}^{j} \eta_{i, j}^{*}\right]<\infty, \quad t \in \mathcal{T}_{n}^{+} .
\end{aligned}
$$

In order to prove (3.18) and (3.21), it is sufficient to show the following system of inequalities hold:

$$
\begin{aligned}
& \varepsilon A \leqslant 1, \text { where } A:=\sum_{i \in \mathcal{I}_{n}^{+}} \sum_{j=0}^{n}\left(\begin{array}{c}
n \\
j
\end{array}\right) \frac{\Gamma(\alpha)}{\Gamma(\alpha+j)} \tau_{i}^{j} \eta_{i, j}^{*}, \\
& \varepsilon B \leqslant 1, \text { where } B:=\frac{1}{\alpha} \sum_{i \in \mathcal{I}_{n}^{+}}\left[\sum_{j=0}^{n}\left(\begin{array}{c}
n \\
j
\end{array}\right) \frac{\Gamma(\alpha)}{\Gamma(\alpha+j)} \tau_{i}^{j} \eta_{i, j}^{*}+\sum_{j=0}^{n+1}\left(\begin{array}{c}
n+1 \\
j
\end{array}\right) \frac{\Gamma(\alpha)}{\Gamma(\alpha+j-1)} \tau_{i}^{j} \eta_{i, j}^{*}\right] .
\end{aligned}
$$

If $\max \{A, B\} \leqslant 1$, then each $\varepsilon \leqslant 1$ would work. Otherwise if $\max \{A, B\}>1$, we choose $\varepsilon=(\max \{A, B\})^{-1}<1$. In any case we are guaranteed of the existence of such $0 \leqslant \varepsilon \leqslant 1$. We state these observations in the following theorem:

Theorem 3.1. There exists no $\alpha>1 / 2$ and $n \geqslant 2$ such that Khabibullin's conjecture is true.

The proof follows the analysis in Sections 3.1-3.3.

\section{Concluding Remarks}

In this work we constructively showed that for any $\alpha>1 / 2$ and $n \geqslant 2, n \in \mathbb{N}$, there exists a nonnegative increasing function $h$ violating the conjecture. The method of construction relied strongly on the monotonicity of the higher order derivatives of the function $\phi$ over $\mathbb{R}^{+}$. In fact, it is the nonemptiness of the set $\mathcal{T}_{\phi(n)}$ for any $n \geqslant 2, n \in \mathbb{N}$ that opens up the way of defining a pair of functions $\{f, h\}$ that fulfils the task. Another key result implied by Theorem 3.1 relates to the question in Bërdëllima [2] of whether the inverse Laplace transform of the function $\phi(z)$ is everywhere nonnegative over $\mathbb{R}^{+}$for some $\alpha>1 / 2$ and $2 \alpha \in \mathbb{N}$. Notice that here $\phi$ is extended to the complex plane, $\phi(z)=\left(1+z^{2 \alpha}\right)^{-1}$ for $z \in \mathbb{C}$. If $\Phi(t):=\left(\mathcal{L}^{-1} \phi(z)\right)(t)$ denotes the inverse Laplace transform of $\phi$, then one can show that

$$
\Phi(t)=\frac{1}{2 \pi i} \int_{\gamma-i \infty}^{\gamma+i \infty} \phi(z) e^{z t} d z=\sum_{k \in \mathbb{Z}_{2 \alpha}} \frac{\exp \left(z_{k} t\right)}{\prod_{k \neq m}\left(z_{k}-z_{m}\right)}
$$

where $z_{k}=\exp ((2 k+1) \pi i / 2 \alpha)$ and $\mathbb{Z}_{2 \alpha}=\{0,1, \ldots, 2 \alpha-1\}$. Indeed, formula in (4.1) follows the Cauchy residue theorem applied on a halfdisk contour around the origin enclosing all poles (singularities) of $\phi(z)$ in the complex plane. The line integral in (4.1) would work for any $\gamma \geqslant 1$ since all poles of $\phi(z)$ lie on the unit circle and $z_{k} \neq 1$ for any $k \in \mathbb{Z}_{2 \alpha}$. In fact, we can establish a more general result that $\Phi(t)$ in (4.1) is not of constant sign on $\mathbb{R}^{+}$. If for some $2 \alpha \in \mathbb{N}$ we had $\Phi(t) \geqslant 0$ for all $t \in \mathbb{R}^{+}$(or $\Phi(t) \leqslant 0$ for all $t \in \mathbb{R}^{+}$), then by theorem 6 in Bërdëllima [2] it would follow that Khabibullin's conjecture would be valid for all nonnegative increasing functions $h$. However, since $\alpha>1 / 2$, this contradicts Theorem 3.1 of this paper which always guarantees a counterexample. Therefore, (4.1) cannot be of constant sign everywhere on $\mathbb{R}^{+}$ 
for any $2 \alpha \in \mathbb{N}$. It must be pointed out that finding sharp estimates when $n \geqslant 2$ and $\alpha>1 / 2$ is still an open problem. Sharipov [8] showed that for each $n \geqslant 2$ and $\alpha>0$ there exists a positive constant $C_{K h}(n, \alpha)$, which is a sharp upper bound for the integral inequality (1.3). Note that as $0 \leqslant \alpha \leqslant 1 / 2$, we have

$$
C_{K h}(n, \alpha)=\frac{\pi}{2} \prod_{k=1}^{n-1}\left(1+\frac{\alpha}{k}\right)
$$

It is possible to numerically bound Khabibullin's constants $C_{K h}(n, \alpha)$ but finding them explicitly as $\alpha>1 / 2$ is yet unsolved. It would certainly be desirable to get, if not in a closed form, at least some explicit formula for $C_{K h}(n, \alpha)$. These constants have implications in estimating the growth rate of a plurisubharmonic function.

\section{A. TeChnical Details}

Lemma A.1. Let $\phi(t)=\frac{1}{1+t^{2 \alpha}}$, then for all $m \geqslant 1, m \in \mathbb{Z}$ we have

$$
\frac{d^{m} \phi}{d t^{m}}=\frac{P\left(t^{\alpha}\right)}{t^{m} \cdot\left[Q\left(t^{\alpha}\right)\right]^{m+1}},
$$

where $Q\left(t^{\alpha}\right)=1+t^{2 \alpha}$ and $P$ is a polynomial function in $t^{\alpha}$ of degree $2 m$.

Proof. We prove this lemma using mathematical induction. For $m=1$ we have

$$
\phi^{\prime}(t)=\frac{-2 \alpha t^{2 \alpha}}{t\left(1+t^{2 \alpha}\right)^{2}}=\frac{P\left(t^{\alpha}\right)}{t \cdot\left[Q\left(t^{\alpha}\right)\right]^{2}},
$$

where $P\left(t^{\alpha}\right)=-2 \alpha t^{2 \alpha}$ as a polynomial function in $t^{\alpha}$ is of degree 2 . Let the statement be true for $m=n$, that is

$$
\phi^{(n)}(t)=\frac{P\left(t^{\alpha}\right)}{t^{n} \cdot\left[Q\left(t^{\alpha}\right)\right]^{n+1}},
$$

where $P$ is a polynomial function in $t^{\alpha}$ of degree $2 n$. Differentiating both sides of the last equation with respect to $t$, we obtain

$$
\frac{d}{d t} \phi^{(n)}(t)=\frac{d}{d t}\left[\frac{P\left(t^{\alpha}\right)}{t^{n} \cdot\left[Q\left(t^{\alpha}\right)\right]^{n+1}}\right] \Leftrightarrow \phi^{(n+1)}(t)=\frac{\alpha t^{\alpha} P^{\prime} Q-n P Q-(n+1) t^{\alpha} P Q^{\prime}}{t^{n+1}\left[Q\left(t^{\alpha}\right)\right]^{n+2}} .
$$

Denote by $\operatorname{deg} P$ the degree of the polynomial $P$. By the inductive hypothesis, since $\operatorname{deg} P=2 n$, we obtain $\operatorname{deg} P^{\prime}=2 n-1$. We observe that $\operatorname{deg} t^{\alpha}=1$ and by definition of $Q$ we have $\operatorname{deg} Q=2$. Setting $P_{0}=\alpha t^{\alpha} P^{\prime} Q-n P Q-(n+1) t^{\alpha} P Q^{\prime}$ and using $\operatorname{deg} P Q=\operatorname{deg} P+\operatorname{deg} Q$ for any two polynomials $P$ and $Q$, then the degree of polynomial $P_{o}$ is

$$
\begin{aligned}
\operatorname{deg}\left(P_{0}\right) & =\max \left\{\operatorname{deg}\left(t^{\alpha} P^{\prime} Q\right), \operatorname{deg}(P Q), \operatorname{deg}\left(t^{\alpha} P Q^{\prime}\right)\right\} \\
& =\max \{1+2 n-1+2,2 n+2,1+2 n+1\} \\
& =2 n+2=2(n+1)
\end{aligned}
$$

Lemma A.2. Let $h(t)=o\left(t^{2 \alpha}\right)$ as $t \rightarrow \infty$ and suppose $h(t)$ satisfies the inequality (2.1). Then the following limits hold

$$
\lim _{T \rightarrow \infty}\left|\phi^{(k)}(T)\right| \int_{0}^{T} \frac{h(x)}{x}(T-x)^{k} d x=0, \quad k \in \mathbb{Z}^{+} \cup\{0\} .
$$


Proof. First we show for $k=0$. We observe that both $\phi(T)$ and $\int_{0}^{T} \frac{h(x)}{x} d x$ are differentiable functions. It is sufficient to prove that the limit vanishes, when the improper integral $\lim _{T \rightarrow \infty} \int_{0}^{T} \frac{h(x)}{x} d x$ diverges. We get an indeterminate form $\infty / \infty$. On the other hand,since $h(t)=o\left(t^{2 \alpha}\right)$ as $t \rightarrow \infty$, we obtain

$$
\lim _{T \rightarrow \infty} \frac{\frac{d}{d T}\left[\int_{0}^{T} \frac{h(x)}{x} d x\right]}{\frac{d}{d T}\left(1+T^{2 \alpha}\right)}=\frac{1}{2 \alpha} \lim _{T \rightarrow \infty} \frac{h(T)}{T^{2 \alpha}}=0
$$

By L'Hospital's Rule [9] we get

$$
\lim _{T \rightarrow \infty} \phi(T) \int_{0}^{T} \frac{h(x)}{x} d x=\lim _{T \rightarrow \infty} \frac{\int_{0}^{T} \frac{h(x)}{x} d x}{1+T^{2 \alpha}}=\lim _{T \rightarrow \infty} \frac{\frac{d}{d T}\left[\int_{0}^{T} \frac{h(x)}{x} d x\right]}{\frac{d}{d T}\left(1+T^{2 \alpha}\right)}=\frac{1}{2 \alpha} \lim _{T \rightarrow \infty} \frac{h(T)}{T^{2 \alpha}}=0 .
$$

Let $k \geqslant 1$, then from the first integral inequality we obtain

$$
0 \leqslant \int_{0}^{T} \frac{h(x)}{x}(T-x)^{k} d x \leqslant T^{\alpha+k}, \quad 0 \leqslant T<\infty .
$$

On the other hand, by Lemma 1 we have that $\phi(T)=O\left(T^{-k-2 \alpha}\right)$ as $t \rightarrow \infty$. Therefore,

$$
\left|\phi^{(k)}(T)\right| \int_{0}^{T} \frac{h(x)}{x}(T-x)^{k} d x \leqslant O\left(T^{-k-2 \alpha}\right) T^{\alpha+k}=O\left(T^{-\alpha}\right) \rightarrow 0 \quad \text { as } \quad T \rightarrow \infty .
$$

This completes the proof.

Lemma A.3. For all $0 \leqslant \alpha \leqslant 1 / 2$ and $t>0$ the identity holds

$$
\operatorname{sign}\left(\phi^{(n)}(t)\right)= \begin{cases}(-1)^{n} & \text { as } 0<\alpha \leqslant 1 / 2, \quad n \geqslant 0 \\ 0 & \text { as } \alpha=0, \quad n \geqslant 1 \\ 1 & \text { as } \alpha=0, \quad n=0\end{cases}
$$

Proof. We begin with the identity

$$
\frac{d^{m}}{d t^{m}}\left(1+t^{2 \alpha}\right)=2 \alpha(2 \alpha-1)(2 \alpha-2) \ldots(2 \alpha-m+1) t^{2 \alpha-m} .
$$

This implies

$$
\operatorname{sign}\left[\frac{d^{m}}{d t^{m}}\left(1+t^{2 \alpha}\right)\right]=\left\{\begin{array}{llll}
(-1)^{m-1} & \text { as } & 0<\alpha<1 / 2, & m \geqslant 1, \\
0 & \text { as } \alpha \in\{0,1 / 2\}, & m \geqslant 2, \\
0 & \text { as } \alpha=0, & m=1, \\
1 & \text { as } \alpha=1 / 2, & m=1, \\
1 & \text { as } \alpha \geqslant 0, & m=0,
\end{array}\right.
$$

for all $t>0$ and $m \in \mathbb{Z}$. We will consider the cases $\alpha=0$ and $\alpha=1 / 2$ separately. Assume that $0<\alpha<1 / 2$. This reduces to showing that

$$
\operatorname{sign}\left(\phi^{(n)}(t)\right)=(-1)^{n}
$$

for all $n \geqslant 0$ and $t>0$. We prove this statement by a strong mathematical induction. The base case would be for $n=0$. By the definition of $\phi(t)$ we have

$$
\left(1+t^{2 \alpha}\right) \phi(t)=1 \Rightarrow \operatorname{sign}\left(\left(1+t^{2 \alpha}\right) \phi(t)\right)=\operatorname{sign}(1)
$$

Since $1+t^{2 \alpha}>0$ for all $t>0$, then $\operatorname{sign}\left(1+t^{2 \alpha}\right)=1$. Thus, we obtain

$$
\operatorname{sign}(\phi(t))=1=(-1)^{0}
$$


Therefore, the base case is true. Assume that the equation $\operatorname{sign}\left(\phi^{(n)}(t)\right)=(-1)^{n}$ is valid for all $n=1,2, . ., k$. Now let $n=k+1$. Differentiating $\left(1+t^{2 \alpha}\right) \phi(t)$ successively $k+1$ times and using Leibniz's formula, we get:

$$
\frac{d^{k+1}}{d t^{k+1}}\left[\left(1+t^{2 \alpha}\right) \phi(t)\right]=\sum_{i=0}^{k+1}\left(\begin{array}{c}
k+1 \\
i
\end{array}\right) \frac{d^{i}}{d t^{i}}\left(1+t^{2 \alpha}\right) \frac{d^{k+1-i}}{d t^{k+1-i}} \phi(t) .
$$

Since $\left(1+t^{2 \alpha}\right) \phi(t)=1$ for all $t \geqslant 0$, then we obtain

$$
\sum_{i=0}^{k+1}\left(\begin{array}{c}
k+1 \\
i
\end{array}\right) \frac{d^{i}}{d t^{i}}\left(1+t^{2 \alpha}\right) \frac{d^{k+1-i}}{d t^{k+1-i}} \phi(t)=0
$$

By the latter equation we arrive at

$$
\operatorname{sign}\left[\left(1+t^{2 \alpha}\right) \frac{d^{k+1}}{d t^{k+1}} \phi(t)\right]=(-1) \operatorname{sign}\left[\sum_{i=1}^{k+1}\left(\begin{array}{c}
k+1 \\
i
\end{array}\right) \frac{d^{i}}{d t^{i}}\left(1+t^{2 \alpha}\right) \frac{d^{k+1-i}}{d t^{k+1-i}} \phi(t)\right]
$$

For $1 \leqslant i \leqslant k+1$ the sign of each summand in $(\mathrm{A} .2$ can be computed as follows:

$$
\operatorname{sign}\left[\frac{d^{i}}{d t^{i}}\left(1+t^{2 \alpha}\right) \frac{d^{k+1-i}}{d t^{k+1-i}} \phi(t)\right]=\operatorname{sign}\left[\frac{d^{i}}{d t^{i}}\left(1+t^{2 \alpha}\right)\right] \operatorname{sign}\left[\frac{d^{k+1-i}}{d t^{k+1-i}} \phi(t)\right]
$$

By A.1 we have

$$
\operatorname{sign}\left[\frac{d^{i}}{d t^{i}}\left(1+t^{2 \alpha}\right)\right]=(-1)^{i-1}
$$

for all $i=1,2, \ldots, k+1$ and $t>0$. On the other side, applying strong induction for $i=$ $1,2, \ldots, k$ on $\operatorname{sign}\left(\phi^{(k+1-i)}(t)\right)$, we get

$$
\operatorname{sign}\left[\frac{d^{k+1-i}}{d t^{k+1-i}} \phi(t)\right]=(-1)^{k+1-i}
$$

Therefore,

$$
\operatorname{sign}\left[\frac{d^{i}}{d t^{i}}\left(1+t^{2 \alpha}\right) \frac{d^{k+1-i}}{d t^{k+1-i}} \phi(t)\right]=(-1)^{i-1}(-1)^{k+1-i}=(-1)^{k}
$$

for all $i=1,2, \ldots, k$ and $t>0$. For $i=k+1$ we have

$\operatorname{sign}\left[\frac{d^{k+1}}{d t^{k+1}}\left(1+t^{2 \alpha}\right) \frac{d^{k+1-(k+1)}}{d t^{k+1-(k+1)}} \phi(t)\right]=\operatorname{sign}\left[\frac{d^{k+1}}{d t^{k+1}}\left(1+t^{2 \alpha}\right) \cdot \phi(t)\right]=(-1)^{k+1-1}(-1)^{0}=(-1)^{k}$.

This shows that all terms A.2 are of the same sign $(-1)^{k}$ for all $i=1,2, \ldots, k+1$ and therefore, we are led to the conclusion that

$$
\operatorname{sign}\left[\left(1+t^{2 \alpha}\right) \frac{d^{k+1}}{d t^{k+1}} \phi(t)\right]=(-1) \cdot(-1)^{k} \Rightarrow \operatorname{sign}\left[\frac{d^{k+1}}{d t^{k+1}} \phi(t)\right]=(-1)^{k+1}
$$

This proves the statement for all $0<\alpha<1 / 2$.

Now let $\alpha=0$. Then $\phi(t)=1$ and this implies $\operatorname{sign}(\phi(t))=1$ and $\operatorname{sign}\left(\phi^{(n)}(t)\right)=0$ for all $n \geqslant 1$ (since $\phi(t)$ in this case is a constant function, its derivatives of all orders vanish). As $\alpha=1 / 2$, we need to prove again that $\operatorname{sign}\left(\phi^{(n)}(t)\right)=(-1)^{n}$ for all $n \geqslant 0$. We need only to use the mathematical induction. For the base case $n=0$ we have

$$
(1+t) \phi(t)=1 \Rightarrow \operatorname{sign}(\phi(t))=1=(-1)^{0}
$$

for all $t>0$. Suppose the statement is true for $n=k$, that is $\operatorname{sign}\left(\phi^{(k)}(t)\right)=(-1)^{k}$. Let $n=k+1$. Differentiating $(1+t) \phi(t)$ successively $k+1$ times, we obtain

$$
\frac{d^{k+1}}{d t^{k+1}}[(1+t) \phi(t)]=\sum_{i=0}^{k+1}\left(\begin{array}{c}
k+1 \\
i
\end{array}\right) \frac{d^{i}}{d t^{i}}(1+t) \frac{d^{k+1-i}}{d t^{k+1-i}} \phi(t)=(1+t) \frac{d^{k+1}}{d t^{k+1}} \phi(t)+\left(\begin{array}{c}
k+1 \\
1
\end{array}\right) \frac{d^{k}}{d t^{k}} \phi(t) .
$$


Since $(1+t) \phi(t)=1$, by definition of $\phi(t)$, we necessarily have

$$
(1+t) \frac{d^{k+1}}{d t^{k+1}} \phi(t)=-\left(\begin{array}{c}
k+1 \\
1
\end{array}\right) \frac{d^{k}}{d t^{k}} \phi(t) \Rightarrow \operatorname{sign}\left[\frac{d^{k+1}}{d t^{k+1}} \phi(t)\right]=(-1) \cdot \operatorname{sign}\left[\frac{d^{k}}{d t^{k}} \phi(t)\right]=(-1)^{k+1}
$$

where in the last step we have used the inductive hypothesis. This completes the proof.

I am grateful to Prof. Dr. R. Luke and our scientific group for making it possible to organize a seminar at our Institute on this mathematical problem. Also I would like to thank the anonymous referee for his/her useful comments in improving the exposition of the paper.

\section{BIBLIOGRAPHY}

1. R. A. Baladai, B. N. Khabibullin. Three equivalent hypotheses on estimation of integrals // Ufa Math. Journal 2:3, 31-38 (2010).

2. A. Bërdëllima. About a Conjecture Regarding Plurisubharmonic Functions // Ufa Math. J. 7:4, 154-165 (2015).

3. N.V. Govorov. Paley's conjecture // Funct. Anal. Appl, 3: 2, 115-118 (1969).

4. B. Khabibullin. Paley problem for plurisubharmonic functions of finite lower order // Sb. Math. 190:2, 145-157 (1999).

5. B. Khabibullin. A conjecture on some estimates for integrals // Preprint. arXiv:1005.3913 (2010).

6. B. Khabibulin. The representation of a meromorphic function as the quotient of entire functions and Paley problem in $\mathbb{C}_{n}$ : survey of some results// Math. Phys. Anal. Geom. 9:2, 146-167 (2002).

7. R. A. Sharipov. A note on Khabibullin's conjecture for integral inequalities // Preprint. arXiv:1008.0376 (2010).

8. R. A. Sharipov. A counterexample to Khabibullin's conjecture for integral inequalities // Ufa Math. J. 2:4, 98-106 (2010).

9. J. Stewart. Calculus: Early Transcendentals. Cengage Learning, Boston (2016).

Arian Bërdëllima,

Institute for numerical and applied mathematics,

University of Göttingen,

37083 Göttingen, Germany

E-mail: arian.berdellima@mathematik.uni-goettingen.de

berdellima@gmail.com 